\title{
La Psicología Social Comunitaria en América Latina como Psicología Social Crítica
}

\section{Latinamerican Community Social Psychology as Critical Social Psychology}

Bernardo Jiménez-Domínguez ${ }^{1}$

\begin{abstract}
Resumen
En este artículo se describe el proceso de desarrollo de la psicología social comunitaria. El inicio coincide con al crítica al modelo médico y las insuficiencias de la psicoterapia y sobretodo de la psiquiatría con respecto a la solución de los problemas psicológicos en el marco del replanteamiento de la relevancia de las ciencias sociales en la explicación de la realidad latinoamericana y el compromiso con la solución de sus problemas.

Esto coincide con el periodo de la crisis de la psicología social dominante. La respuesta a dicha crisis es una psicología crítica reinsertada en el campo de las ciencias sociales y de las perspectivas cualitativas, críticas rechazadas por el peso de la psicología social vigente. De estos desarrollos se deriva la relación con la emergencia internacional de una psicología social crítica y la ubicación de la psicología social comunitaria latinoamericana como el principal aporte a dicha perspectiva desde el continente.
\end{abstract}

Palabras claves: Psicología comunitaria social crítica

\begin{abstract}
The process of development of community social psychology is described. This process coincides with a criticism of the medical model, as well the insufficiency of the psychiatric and psychotherapeutic solutions to psychological problems in the framework of a restatement of the relevance of the Social Sciences in the understanding of Latin-American reality and the commitment with a solution of these problems.

This happens simultaneously with the crisis of mainstream social psychology. The answer to such a crisis is a psychology reinserted in the field of social sciences and the qualitative, critical and forgotten perspectives denied by mainstream psychology. From these developments relationships with the international emergence of a critical social psychology are established Community social psychology of Latin America is considered a main contribution in this perspective.
\end{abstract}

Key words: mainstream critical community psychology.

1 Psicólogo, Centro de Estudios Urbanos, Universidad de Guadalajara ceur@cencar.udg.mx 


\section{Introducción}

A través de una revisión histórica del nacimiento de la psicología social comunitaria y de la psicología comunitaria, en este artículo se invita a examinar algunos de los principales conceptos y orientaciones que han dado fundamento a su quehacer. Para esto se hace necesario reconocer sus características históricas, sus implicancias prácticas y las construcciones teóricas desarrolladas desde su inicio.

Más que un texto que invite a obtener certezas disciplinarias, lo que pretende es realizar un acercamiento a la psicología social comunitaria desde una visión crítica. De manera de promover a la continua reflexión y construcción de este ámbito de la psicología.

\section{Historias Diversas y Diversas Psicologías}

Este artículo busca rastrear brevemente algunas de las historias menos citadas detrás de la denominada psicología comunitaria y la psicología social comunitaria y revisar algunos de los desarrollos recientes en este campo y en general en las ciencias sociales cercanas, que sirven como parte del esquema alternativo dentro del paradigma de la acción y el giro participativo.

Cuando se hace la historia de la Psicología Social Comunitaria se suelen mezclar historias diversas e identificar la psicología comunitaria, que surgió en los EEUU en condiciones sociales, políticas y académicas bien definidas, con la psicología social comunitaria, que si bien puede estar relacionada de alguna forma, se debe considerar como algo específicamente derivado de la psicología social latinoamericana en sus varias vertientes. Se las equipara al usar los términos indistintamente y al decir en el mismo renglón, psicología comunitaria o psicología social comunitaria, como en la reseña introductoria del libro de Sánchez y Wiesenfeld (1995). En las compilaciones sobre el tema suelen igualmente aparecer trabajos que tienen orígenes muy diversos, pero presentados como si formaran parte de lo mismo y sumados dieran una psicología social comunitaria latinoamericana. Una autora que establece algunas diferencias, con base en el conocido esquema de la antropología en o de la ciudad, es Freitas (1996), quien dice que la psicología en la comunidad recibe dicha identidad de la comunidad misma y se relaciona con el intento de deselitizar la psicología y vincularla a la realidad brasilera en los años 60 y 70. La psicología de la comunidad se ubica en las dos décadas siguientes como el esfuerzo de llevar los servicios de salud a la comunidad con instrumentos derivados de la vertientes clínicas y educativas. Mientras que la psicología social comunitaria se encuadra en la psicología social, redefinida en función del trabajo con grupos para la formación de la conciencia crítica y la construcción de una identidad social basada en una ética humanista.

En 1981, el ya difunto sociólogo mexicano Francisco Gomezjara, señalaba como un hecho curioso el que en cada ciclo de crisis económica aparezcan programas de desarrollo comunitario con un cariz académico, humanista y con pretensiones técnico-científicas: cuando el movimiento campesino se insurrecciona, cuando los países desarrollados tienen un superávit de capital y mercancías para negociarlas bajo la forma de ayuda a los países del tercer mundo, cuando la migración campesina aumenta por la industrialización agrícola y por el hambre, cuando las comunidades exigen servicios y se autoorganizan. La autoorganización solía ser la señal que anunciaba la llegada eventual de programas de desarrollo comunitario desde los años 60. Como bien lo explica Statman (1974), los movimientos populares primero son reprimidos por la fuerza y luego sepultados en un diluvio de programas comunitarios, dichos programas no suelen resolver casi nada pero tranquilizan a la gente por un rato con las promesas del cambio. La sumisión se logra por medios no coercitivos y para ello resultan útiles los psiquiatras, psicólogos, 
educadores, trabajadores sociales, estudiantes universitarios en prácticas obligatorias y otros agentes de cambio social planificado y por lo general bien intencionado desde diversas instituciones externas a la comunidad. En tiempos globalizados, muchos de estos esfuerzos se ahorran porque la dualización invisibiliza los problemas y las comunidades y dichos programas solo aparecen en tiempos preelectorales como una teatralización cuyo escenario se recoge poco después de la función porque lo prioritario sigue siendo la macroeconomía y las ilusiones vanas de la inversión extranjera.

Se suele citar como la fecha fundacional de la Psicología comunitaria el año 1965 y la Conferencia de Swamppscott (Conference on the Education of Psychologists for Community Mental Health), en la que se cuestiona el carácter individual de la intervención y se plantea el nivel de lo comunitario como el mas adecuado. Pero no se acostumbra citar el antecedente inmediato de la aparición de una psiquiatría y psicología comunitarias en EEUU durante el gobierno de Kennedy relacionada con la Community Mental Health Center Act de 1963, dirigida a resolver problemas de presupuesto a nivel institucional y de paso, pacificar a las poblaciones mas empobrecidas del país (para América Latina tenían la Alianza para el Progreso y los cuerpos de paz), implementado con ayuda de profesionales de la salud mental y a través de los Centros de salud Mental Comunitaria que buscaban proporcionar servicios de hospitalización, tratamiento ambulatorio, urgencias, hospitalización parcial, programas educativos y asesoría a instituciones. El objetivo planteado por el mismo Kennedy en su discurso del 31 de octubre de 1963 era que los costosos, inefectivos y en ese momento muy cuestionados hospitales psiquiátricos fueran sustituidos por estos centros más económicos y operativos. De hecho, la población de pacientes se redujo de 558 mil en 1955 a 193 mil en 1973. ¿Pero cuáles son los factores adicionales que están detrás del decreto presidencial? Según diversos autores (Nietzel, 1979, Castel y Lovell,1980,
Jiménez, 1983) algunos de los asuntos claves que confluyen son:

1. Las denuncias y críticas desde muy diversos frentes en contra de los Hospitales Psiquiátricos, las psicoterapias, el modelo médico en este campo (con su énfasis clínico en factores intrapsíquicos) y la rotulación estigmatizante derivada de las pruebas psicológicas, aumentan y encarnan en el público a través de los medios.

2. Aparecen los psicotrópicos y se comienzan a poner en práctica alternativas grupales, comunitarias, se replantea el entrenamiento de los psicólogos clínicos y psiquiatras y aparecen experiencias de desinstitucionalización psiquiátrica en Europa y movimientos antipsiquiátricos y de psiquiatrizados y en contra de prácticas ampliamente criticadas y que atentan contra los derechos de los pacientes, como el electroshock y la psicocirugía.

3. A finales de los años 70 había medio millón de pacientes de mas en los Hospitales Psiquiátricos de EEUU que afectaban los presupuestos estatales y el costo de construcción de dichas instituciones de encierro resultaba muy elevado y su eficiencia nula.

Una de las consecuencias de los nuevos programas comunitarios consiste en generar una apariencia de desinstitucionalización, que en realidad era más bien cooptación de la participación comunitaria. Encubría además un negocio por parte de empresas privadas que administraban los hoteles de paso para ex pacientes antes de supuestamente reinsertarse en la comunidad, que fueron un enorme fracaso pero un gran negocio. Ello da lugar a una política de Puerta Giratoria (revolving door), a través de la cual los pacientes que eran echados a la calle (rememorando prácticas medievales), volvían a ingresar cíclicamente. Esta psiquiatría-psicología comunitaria inicia desinstitucionalizando el problema de la enfermedad mental para pa- 
sar a institucionalizar los problemas que le dan origen en la comunidad. Lo cual se inspira en algo muy estadounidense, la community participation claramente ligada a la forma de trabajo social de los grupos protestantes para evangelizar. Además, como lo explica Gondra (1977), el mismo término comunidad cumple una función ambigua gracias a la multiplicidad de significados que se le pueden atribuir, cuando se habla de participación comunitaria significando colaboración con el gobierno. Cuando se define un sector geográfico caracterizado por la miseria derivada del anárquico y desigual crecimiento urbano y se lo rotula como comunidad para aislarlo en su propia dinámica y justificar la ayuda externa. Los estudios de comunidad siempre se refieren a ella como totalidad, fragmento de la misma o sitio de los acontecimientos. Un cuestionamiento adicional al concepto de comunidad, es el que hace Young (1990), al plantear que sería mejor adoptar la ciudad misma, mas que la comunidad como ideal de justicia, porque el ideal de comunidad está construido sobre criterios de una supuesta igualdad o autosuficiencia, mas que sobre las grandes diversidades que constituyen el espacio urbano. La vida de la ciudad ofrece diferencias y si la política urbana se plantea democráticamente todos los grupos que la habitan deben ser tomados en cuenta y tener voz sin que necesariamente conformen una comunidad.

\section{La Diferencia desde el Sur}

Si pasamos a Latinoamérica podemos citar otra historia, también ligada al activismo comunitario religioso, pero en un contexto de definición muy diferente. Santiago, Serrano y Perfecto (1983) describen la aparición de una psicología social comunitaria en Puerto Rico, que vinculaban por una comunalidad asumida con la religión cristiana. Desde la psicología social partían de sus limitaciones tan cuestionadas en el conocido periodo de la crisis de la disciplina y que ellos resumían en su orientación positivista, ahistórica y ateórica, aislada de las demás ciencias sociales y el énfasis individualista de su definición. Para ellos lo social comunitario implicaba un marco interdisciplinario comprometido con la acción social. Teóricamente citaban el construccionismo de Berger y Luckman y a Moscovici y definían el rol del psicólogo social comunitario como un facilitador, concientizador y promotor de un cambio social que debe ser coordinado, planificado e implementado por la misma comunidad.

Por otro lado, en el plano religioso se apoyaban en los principios de la teología de la liberación y su compromiso con el pensamiento transformador y las luchas de los oprimidos en el continente latinoamericano y citaban una serie de aspectos comunes entre esta y la psicología social comunitaria alrededor de la metodología participativa y la búsqueda de la justicia y la solidaridad como auto investigación y autogestión, a partir también de la problematización planteada por Freire. Era claro en esta propuesta que lo interdisciplinario y el compromiso social no suprimían el nivel específico de un nuevo rol profesional para el psicólogo social y que se planteaba como una alternativa al rol tradicional cientificista de neutralidad valorativa que asumía como suyos los problemas desde la comunidad y aprendía de su experiencia y conocimiento local. Esta propuesta de intervención en la investigación (Irizarry y Serrano García, 1979) ha tenido un desarrollo muy original y se ha cualificado hasta convertirse en programa de doctorado y diversificado hasta plantear mas recientemente, un modelo de psicología social comunitaria en el campo de la salud (Serrano, Bravo, Rosario y Gorrín, 1998). Dicho modelo parte de la crítica al modelo biomédico desde un enfoque biopsicosocial, en el que la enfermedad es vista como un desbalance no solo de lo biológico sino también de aspectos sociales, económicos, culturales y espirituales y abogan por el poder de sanación del cuerpo y la reducción al mínimo de los medicamentos y los medios fisicoquímicos de los que deviene el poder médico y sobretodo psiquiátrico. 


\section{Una Evaluación Crítica y una Alternativa}

Sin embargo, en un libro que recoge la experiencia 10 años después ( Serrano y Rosario,1992), una destacada psicóloga social ya fallecida, María Milagros López, cuestionaba críticamente una serie de aspectos derivados de las contradicciones entre un discurso radical sobre la autonomía de las comunidades y una práctica que comenzó a depender de los servicios del Estado y a institucionalizarse, la práctica seguía siendo además una extensión de las prácticas universitarias y el énfasis puesto en la prevención tiende a confundirse con la ausencia de conflicto, razón por la cual demanda cuestionar la noción de intervención. Lo que resulta de interés en esta crítica desde dentro, es el poner en práctica, lo que constituye una de las características del saber de las ciencias sociales y en este caso, de la psicología social, mas si esta se define desde un contexto alternativo y crítico. Me refiero a la provisionalidad del saber en ciencias sociales por su carácter intrínsecamente histórico y a la necesidad, tal como lo hace López en la crítica citada, de estar deconstruyendo los saberes y las prácticas. La imagen que usa Ibáñez (1994) para ilustrar este proceso es el de Penélope, tejer y destejer como una rutina necesaria, definirse como un constructor de obras efímeras. Según él, la psicología social crítica debe definirse en términos de nuevas prácticas, que debiliten los efectos de poder del objetivismo, pero sin reproducir sus estructuras de dominación. O como lo planteaba Heather (1976) al proponer la psicología social como una ciencia de las personas genuinamente reflexiva: no debe ser una ciencia hecha por alguna gente, los expertos, sobre otra gente, los no expertos, para el beneficio de otra gente, los que tienen el poder de emplear a los psicólogos. Ibáñez (1997) dice a ese respecto, que una forma de definir la psicología social es precisamente, como la actividad de una comunidad de trabajadores pagados por diversas instituciones. Siendo esta condición de asalariados más importante de lo que se cree en la conformación de los rituales meritocráticos que pueden limitar la condición crítica y las prácticas derivadas de la psicología social. La psicología social crítica en la que podríamos encuadrar a la psicología social comunitaria latinoamericana así planteada, se puede resumir en tres características, deconstrucción, pensamiento crítico y transdisciplinariedad y el estar situada simultáneamente en el plano conceptual y en la esfera práctica.

\section{El Paradigma de la Acción}

Este último dilema está claramente resuelto en la orientación mas conocida de la psicología social comunitaria, que es la que se ubica en el marco de la redefinición comprometida y política con la justicia social del aporte lewiniano de la investigación acción transformado en Latinoamérica en el paradigma de la acción, la investigación acción participativa. Hay que decir que la propuesta de Lewin fue un buen intento de responder el dilema con la simultaneidad teóricopráctica de la investigación acción, resumido en la conocida frase: "no hay nada más práctico que una buena teoría". Pero por el peso de la psicología dominante alejada del contexto de desarrollo de las ciencias sociales su redefinición no fue hecha dentro de la psicología social, sino por fuera de ella, en la sociología militante de los años 60, en un contexto de influencia del marxismo revolucionario, las teorías de la dependencia, la filosofía de la liberación y la pedagogía del oprimido. De aquí es de donde por necesidad y también por una interacción transdiciplinaria y un proceso continental de conciencia política, es retomado este nuevo enfoque.

La autora más conocida en esta corriente es Maritza Montero que ha definido una psicología social crítica, una psicología social comunitaria y una psicología de la liberación, aunque suele referirse en general mas a la psicología comunitaria como subdisciplina que incluye a la psicología social comunitaria. 
Montero (1996) ubica varias áreas que según ella comprenden los ámbitos de intervención de la psicología comunitaria: la salud comunitaria, la educación comunitaria, el trabajo comunitario en organizaciones y aquí ubica a la psicología social comunitaria, como una de estas áreas pero que sirve como encuadre de las anteriores, en lo que define como la subdisciplina de la psicología comunitaria. Por psicología social comunitaria entiende el énfasis en el desarrollo comunitario, en la organización popular y en los procesos psicosociales ligados a ellos. En su obra mas reciente (Montero, 2003) publicada por Paidós, dice que el enfoque psicosocial comunitario incluye aspectos ligados a la salud mental y la salud comunitaria bajo la premisa de que los problemas no son de carácter individual sino que residen en las relaciones interpersonales, contextuales y sociales. Ubica su enfoque como parte de una corriente internacional que denomina de construcción y transformación crítica. Los antecedentes que reconoce en Latinoamérica son un enfoque marxiano y marxista, la sociología militante iniciada por Fals Borda y la educación popular planteada por Freire. El método es la Investigación Acción Participativa (IAP) que permite adoptar diferentes enfoques y estrategias metodológicas en la psicología comunitaria. Lo cual es evidente en la evolución misma de Fals Borda, que ha escrito sobre la IAP usando diferentes enfoques teóricos en diferentes momentos durante los últimos 40 años. Por lo general se reconoce su influencia y es considerado como el principal autor en la redefinición crítica de la investigación acción, pero en el medio de la psicología las citas sobre Fals Borda giran alrededor de muy pocas de sus obras y parece desconocerse que él mismo se ha reciclado conceptualmente y hecho balances críticos significativos a lo largo de su vida. Curiosamente, él también tuvo un inicio ligado al desarrollo comunitario de origen religioso, pues era presbiteriano e hijo de una de las dirigentes de esa iglesia y los otros dos intelectuales colombianos con los que formó el grupo La Rosca en 1970, eran ministros ordenados de la misma. Los tres buscaban un método participativo que les permitiera poner la ciencia al servicio de los movimientos populares (Jiménez,1994). Ese método fue tomado coincidencialmente de la psicología social de Kurt Lewin, como investigación acción, lo cual reconoce Fals Borda en una entrevista que le hice en 1991, y esta concepción tiene también una clara relación con la ya citada community participation y el activismo de las iglesias protestantes para la solución local de los problemas comunitarios en los EEUU. Lo que le agrega Fals Borda y el grupo La Rosca al considerarla insuficiente es una teoría y una praxis liberadora usando el concepto de inserción, que implica que el investigador se involucre en el proceso que estudia. Fals Borda reconoce por otro lado que el antecedente disciplinario de la investigación participativa es la tradicional observación participante de la antropología que el replantea inicialmente como observación intervención y se sintetiza en observación inserción bajo la influencia del marxismo dominante en ese momento en las universidades latinoamericanas.

Actualmente la IAP es reconocida mundialmente como un aporte hecho desde el Sur y su influencia se ha generalizado a muy diferentes contextos y espacios. Lo que no se puede decir en el campo de la psicología social comunitaria es que esta orientación que está a la base de su definición crítica haya sido reapropiada y desarrollada mas allá de los planteamientos hechos desde la sociología y específicamente de los aportes de Fals Borda y otros investigadores en el campo de la IAP, básicamente se lo repite y se lo repite de forma limitada. En el último congreso mundial de investigación participativa, Fals Borda (1998) hizo un gran énfasis en su compilación en el nivel de lo participativo, escribiendo incluso la A de acción entre paréntesis. Veamos para terminar algunos aspectos sobre el tema de la participación que al igual que la IAP dio un gran salto y ahora complementa como necesaria al término democracia desde la ciudadanía. 


\section{La Participación es Cuestión de Grados}

La participación es consustancial a la ciudadanía en un contexto en el que, tal como ha venido sucediendo en los últimos 20 años, el concepto de participación se ha popularizado en el discurso político y ya no solo en el nivel comunitario. A partir de la conciencia con sus inclinaciones y expresiones violentas, como señala Fals-Borda (1992), la gente se acostumbró al Estado-nación centralizado como si fuera algo dado y natural, y se ha gastado mucha energía en la construcción de su maquinaría y estructura de poder desde el siglo XVI sin que los resultados sean para nada satisfactorios. Así explica la necesidad de refrenar el violento poder estatal y dar la oportunidad a la sociedad civil, en un esfuerzo participativo de abajo hacia arriba $\mathrm{y}$ de las periferias hacia los centros.

La participación en el aspecto político es el factor que posibilita que los dos supuestos que caracterizan a la democracia, se cumplan: que todas las personas tengan una participación real en el poder y que los gobernan- tes estén sometidos al control efectivo y permanente de los gobernados. La democracia participativa sería aquella en la que la esfera estatal y la social se superponen parcialmente, dando lugar a una intersección que representa el espacio público de la participación (Murillo y Pizano, 1999).

Otra forma de distinguir entre diferentes formas de participación es pensar en términos de los niveles de participación. Estos pueden ser entendidos a lo largo de un continuo y pueden variar de la participación como un acto de manipulación a un nivel en que la gente se hace partícipe en el desarrollo de iniciativas, asumiendo además el control de su manejo. Podríamos ubicar ocho pasos en este continuo, parte de los cuales se relacionan con una perspectiva eficientista que se basa en la economía costo-tiempo. Cuando se va más allá de la información y se adopta una perspectiva de consenso, se puede hablar de una participación auténtica y horizontal. Este continuo lo podemos representar de la siguiente forma:

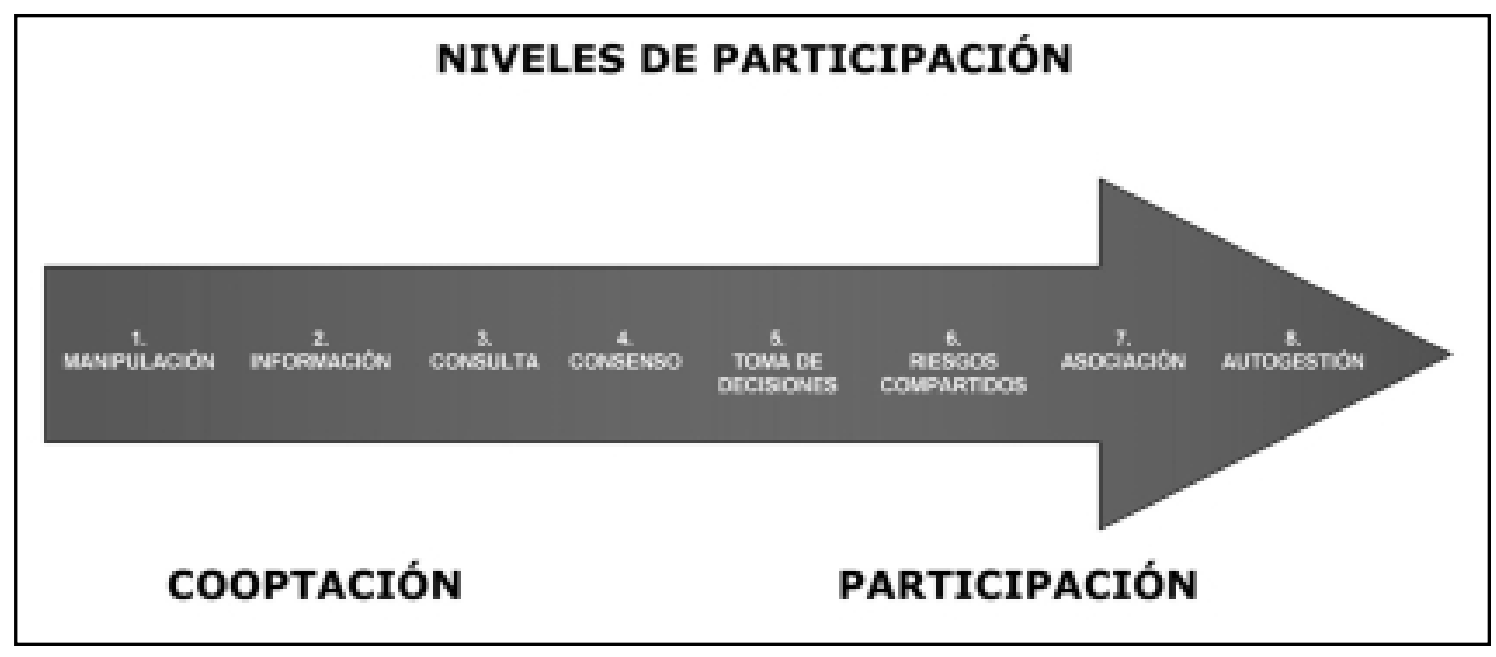




\section{De la Cooptación a la Vivencia}

La participación ciudadana se refiere en concreto al asunto del poder y su ejercicio por parte de diferentes actores sociales en los espacios creados para la interacción entre los ciudadanos y las autoridades locales. Según Gaventa y Valderrama (1999), en América Latina el grado de descentralización no ofrece en general un espacio real para la participación de los movimientos sociales, porque la cuestión del poder no se trata en consecuencia. Lo cual supone en la práctica que las élites y gobiernos locales, así como también los partidos políticos e incluso algunas ONGs y diversos tipos de instituciones que manejan programas de asistencia, investigación e intervención comunitaria (las universidades y sus prácticas obligatorias suelen caer en ello), no tienen problema en cooptar a las comunidades y organizaciones populares con el fin de llevar a cabo sus programas. La única forma de contrarrestar esta situación está directamente relacionada con asesorias solidarias, la experiencia organizacional y la vivencia (Fals-Borda, 1998) en procesos de planificación y acción participativa. Lo cual refiere más específicamente al asunto del conocimiento como poder, al necesario aprendizaje autogestivo de habilidades de negociación política a partir de una movilización efectiva de las organizaciones y comunidades de base para lograr cambios e influencia en las decisiones, que permitan contrarrestar los mecanismos de cooptación. El marco más adecuado para lograr este conocimiento es el de la investigación-acción participativa (IAP) desarrollada en América Latina y otras regiones del Sur, cuyo desarrollo reciente (también expuesto a la cooptación desde el primer mundo) ha sido descrito por Rahman y Fals-Borda (1992).

La cooptación de la participación, ya sea desde el poder, las fundaciones, las universidades o partidos de izquierda y derecha, lleva a que, no todo lo que hoy se llama "participación" sea participativo (Fals-Borda, 1986), o que como lo señala Montero (1996), no siem- pre que se habla de participación, el referente sea un proceso de cooperación, solidaridad, construcción y apropiación del objeto por los actores sociales partícipes. El uso del concepto de manera indefinida, lo convierte según ella, en un paraguas bajo el cual se cobijan diferentes grados de participación que varían en función del vínculo con grupos de base o con organizaciones estatales o no gubernamentales, en función del poder y control que manejen los participantes. La cooptación en la participación se refiere en general al mantenimiento de la asimetría en la interacción. Participar, es según Fals-Borda (1986), el rompimiento voluntario y vivencial de la situación asimétrica de sumisión y dependencia implícita en la relación sujeto-objeto.

La ruptura de la asimetría implica lo que Fals-Borda (1998) llama la vivencia participante horizontal, que permite una relación auténticamente dialógica. Que en el caso de la IAP permite la interpenetración entre el conocimiento popular y el experto a partir de un compromiso existencial que implica una transformación de la personalidad y la cultura, la rebelión contra la rutina, el egoísmo y la manipulación. Esto se discutió en el congreso mundial de IAP en Cartagena en 1997, como la acción necesaria para construir un contrapoder o generar poder popular. En general, como aclara Fals-Borda (1998), toda investigación participativa sería interacción comunicacional en la que ocurre un proceso no solo de diálogo, sino de confianza mutua entre investigador e investigado producida vivencialmente. El compartir la experiencia en un proceso comunitario entre los participantes, está muy bien definida por uno de los entrevistados de Sánchez (2000) al decir que participar "es el sentimiento de que cada cosa pertenece a todos", lo que lo lleva a concluir que la participación genera solidaridad durante el proceso de convivencia de la comunidad.

Si la vivencia como condición de la participación, que sigue también el principio de cooperación de los hablantes, se sitúa localmente, estaríamos hablando de la episteme 
del "habemos" que personaliza la lengua castellana convirtiéndola en pura relación, tal como lo ejemplifica Moreno (1995) al proponer una episteme de la relación que caracteriza el mundo de vida de la episteme popular venezolana y latinoamericana.

Lo que todo esto plantea, es no solo la necesidad de profundizar la participación ciudadana a partir de los movimientos sociales que la representan y a través de alianzas y consensos que las fortalezcan y defiendan de la represión y cooptación desde el poder, sino también la urgencia de un mayor énfasis en la educación ciudadana, en experiencias participativas y aprendizajes liberadores que contribuyan a crear las condiciones y tecnología ciudadana como prácticas que preparen a la ciudadanía y despierten sus interés en la gestión pública como un asunto de todos, tanto a nivel comunitario, social y político en la marcha sin furia (como lo propone Fals-Borda, 1992) hacia una democracia participativa, como también hacia una ciudadanía global. He ahí un programa para la inserción de una psicología social comunitaria como parte de la corriente internacional de la psicología social crítica.

\section{Comentarios Finales}

Tanto la psicología social comunitaria como la psicología comunitaria nacen en contextos históricos y políticos bien definidos, lo que implicó consecuencias directas en todos los actores que se vieron involucrados. Para la detección de estas implicancias es necesario explicitar las diferencias existentes entre ambas corrientes de la psicología.

Esta revisión histórica, que se realiza en el artículo, no sólo permite diferenciar hechos y preguntas como cuándo y dónde surgieron, sino que da claras luces sobre concepciones teóricas y prácticas de cada una de ellas; diferencias que hacen aún más fuerte sus distinciones y particularidades. En este sentido es que el paradigma de la acción y los conceptos de participación marcaron en América Latina una psicología social comunitaria que respondió, y aún intenta responder, a la promoción de un cambio social sostenido en metodología de orden participativo y de justicia social. Esta corriente no implica, solamente un desarrollo académico en esta línea de acción; sino que trae consigo una carga política en cuanto su desarrollo ha implicado prácticas en el orden social.

Como se dijo en un inicio, si bien el texto no intenta obtener certezas, invita a reflexionar sobre la psicología social comunitaria que se está desarrollando actualmente en América Latina. Reflexión que es necesaria hacer desde el reconocimiento de la construcción social que ha implicado esta corriente, alejándola cada vez más de visiones objetivistas propias de los poderes centrales.

En la medida que existan prácticas que intenten responder a las orientaciones de la psicología social comunitaria, es necesario mantener y promover la discusión sobre qué disciplina se construye y sus implicancias. En este sentido, conceptos como la participación invitan a que esta reflexión se realice reconstruyendo prácticas y saberes, que en muchas ocasiones se dan por aceptados.

\section{Referencias}

Castel, F. y Lovell, A. (1980) La sociedad psiquiátrica avanzada. Barcelona: Anagrama

Fals-Borda, O. (1986) Conocimiento y Poder Popular. Bogotá: Siglo XXI y Punta de Lanza.

Fals-Borda, O. (Comp.)(1998) Participación Popular: Retos del Futuro. Bogotá: ICFES, IEPRI y COLCIENCIAS.

FreItAs, M.F. (1996) Psicologia na comunidade, psicologia da comunidade e psicologia social comunitaria: praticas da psicología em comunidade nas décadas de 60 a 90, no Brasil. En Freitas, R.H. Psicología Social Comunitaria. Petrópolis: Editora Vozes. 
Gaventa, J. \& Valderrama, C. (1999) Participation, Citizenship and Local Governance. Ponencia presentada en el Taller: Strengthening participation in local governance. Institute of Development Studies, June 21-24.

GomeZjara, F. (1981) Técnicas de desarrollo comunitario. México: Nueva Sociología.

Gondra, D. (1977) El concepto de comunidad y su relación con los problemas de salud. Educ. Med. Salud. Vol II, 3.

Heather, N. (1976) Radical Perspectives in Psychology. London: Methuen.

IBÁÑEZ, T. (1994) Psicología social construccionista. Guadalajara: Editorial UdG.

IbáÑEZ, T. \& IÑ̃guez, L. (1997) Critical Social Psychology.London: Sage

IRIZARRY, A. y SERRANO, I. (1979) Intervención en la investigación. Avepso Boletín, Vol 2, 3, pp. 6-22.

LÓPEZ, M. M. (1992) Ajuste de cuentas con la psicología social comunitaria: balance a diez años. En Serrano,I. y Rosario, W. Contribuciones puertorriqueñas a la psicología social comunitaria. Rio Piedras: Universidad de Puerto Rico.

Montero, M. (1996) La participación: significado, alcances y límites. En: M. Montero, E. Jaua, E. Hernández, J. P. Wyssenbach, S. Medina, S. Hurtado y A. Janssens, Participación. Ámbitos, retos y perspectivas. Caracas: CESAP.
Montero, M. (1998) La comunidad como objetivo y sujeto de la acción social. En Martín , A. Psicología comunitaria. Madrid: Síntesis.

Montero, M. (2003) Teoría y práctica de la psicología comunitaria. Buenos Aires: Paidós.

Moreno, A. (1995) El aro y la trama. Episteme, modernidad y pueblo. Caracas: CIP.

Murillo, G. y Pizano, L. (1999) La democracia participativa en la encrucijada: el caso colombiano. En: S. Sosnowski y R. Patiño (Comps.) Una cultura para la democracia en América Latina. México: FCE y UNESCO.

Nietzel, M. T. (1979) Crime and its modification: a social learning analysis perspective. New York: Pergamon Press

Sánchez,E. (2000) Todos con la "Esperanza". Continuidad de la Participación Comunitaria. Caracas:UCV.

Sánchez, E. y Wiesenfeld, E. (1995) La psicología social comunitaria en América Latina. En Wiesenfeld, E. Y Sánchez,E. Psicología social comunitaria. Caracas: Tropykos.

Santiago, L. Serrano,I. y Perfecto, G. (1983) La psicología social comunitaria y la teología de la liberación. Avepso Boletín, Vol 6,1, pp. 15-22.

Statman, J. (1974) La salud mental de la comunidad como programa de pacificación. En Agel, J. El terapeuta radical. México: Extemporáneos.

Young, I.M. (1990) Justice and the Politics of Difference. Princeton: University Press

Fecha Recepción Artículo: 08 de Agosto 2003

Fecha Evaluación Final: $\quad 29$ de Octubre 2003 